\title{
A Simple Theory of Argument Schemes
}

\author{
G.C. GODDU
}

Department of Philosophy

University of Richmond

401 Ryland Hall

Richmond, Virginia

USA

ggoddu@richmond.edu

\begin{abstract}
While there has been in depth discussion of many particular argumentation schemes, some lament that there is little to no theory underpinning the notion of an argumentation scheme. Here I shall argue against the utility of argument schemes, at least as a fundamental part of a complete theory of arguments. I shall also present and defend a minimalist theory of their nature-a scheme is just a set of proposition expressions and propositional functions. While simple, the theory contravenes several typical desiderata of argumentation schemes such as (i) aiding in the identification of enthymemes and (ii) keeping arguments constrained to a manageable taxonomy. So much the worse for the desiderata. Instead, I shall recommend focusing less on schemes and more on the component propositional functions.
\end{abstract}

Résumé: Bien qu'il y ait eu une discussion approfondie sur de nombreux schèmes d'argumentation particuliers, certains déplorent qu'il y ait peu ou pas de théorie sous-tendant la notion de schème d'argumentation. Ici, je vais avancer des arguments contre l'utilité des schèmes d'argumentation, au moins en tant que partie fondamentale d'une théorie complète des arguments. Je présenterai et défendrai également une théorie minimaliste de leur nature: un schème n'est qu'un ensemble d'expressions de proposition et de fonctions propositionnelles. Bien que simple, la théorie enfreint plusieurs desiderata typiques des schèmes d'argumentation tels qu'aider à (i) identifier des enthymèmes et (ii) limiter les arguments à une taxonomie gérable. Tant pis pour les desiderata. Au lieu de cela, je recommanderai de concentrer moins sur les schèmes et plus sur composants des propositions fonctionnelles.

Keywords: argumentative constant, argumentative force, argument taxonomy, critical questions, propositional functions, schemes 


\section{Introduction}

Even though there has been in depth discussion of many particular argumentation schemes (see, for example, Macagno and Walton 2017; Walton 2000; Walton 1999; Walton 1996), there has also been a lament that there is little to no theory underpinning the notion of an argumentation scheme (Dove and Nussbaum 2018). Here I shall present and discuss the consequences of some very minimalist theories of what argumentation schemes are.

I emphasize that my focus here is on the nature of argumentation schemes and not a general theory of schemes, though, of course, the nature of schemes will have consequences for a more general theory. Without an adequate theory of the nature or ontology of schemes we cannot hope to achieve an acceptable and firmly grounded general theory of schemes. Such a general theory might also include a categorization of schemes, a means of distinguishing good schemes from bad ones, an articulation of how to use schemes to aid in other aspects of argumentation theory, etc. Regardless, I shall only briefly touch on a more general, yet still quite simple, theory of argumentation schemes towards the end of the paper.

In section 2, I shall present an underlying assumption of many theorists about schemes and discuss two minimalist theories of the nature of schemes consistent with that assumption. In section 3 , I shall argue that these two minimalist theories do not satisfy the typical desiderata theorists hope to achieve by appealing to argumentation schemes. In section 4 , I shall suggest that attempts to modify the theories, or to reject the underlying assumption, to meet the desiderata are unlikely to succeed. Finally, in section 5, I shall hypothesize that instead of taking the results of section 3 and 4 as a reductio of the minimalist theories (or the underlying assumption), we should instead focus less on schemes and more on the component parts.

\section{What are argument schemes?}

According to Douglas Walton, Chris Reed, and Fabrizio Macagno, in their book, Argumentation Schemes: 
Argumentation schemes are forms of argument (structures of inference) that represent structures of common types of arguments used in everyday discourse, as well as in special contexts like those of legal argumentation and scientific argumentation (2008, $\mathrm{p}, 1){ }^{1}$

Though I am not sure what it means for a form of argument to represent a structure of a common type of argument, we can start with the notion that argument schemes are at the very least forms of argument. Indeed, their very next sentence is: "They include the deductive and inductive forms of argument that we are already so familiar with in logic ..." (Walton, Reed, and Macagno, 2008, p. 1; see also, p. 12). Though perhaps not wholly explicit, Christoph Lumer (2011), David Hitchcock, (2017), and Manfred Kienpointner, (2018), all, at least by the examples they give, endorse this last claim. Hence, whatever argument schemes are, it is as least true that:

(1) Logical forms are a type of argument scheme.

Here, then, is a simple way to get a theory that accepts (1). Suppose an argument is a set of a set of propositions and another proposition. Argument schemes are the forms of arguments or schematic arguments. Propositional functions are the forms of propositions or schematic propositions. Hence, the simplest theory of the nature of argument schemes would be:

T1: An argument scheme is a set of a set of propositional functions and another propositional function.

\subsection{Propositional functions}

What exactly is a propositional function? According to Russell and Whitehead:

By a 'propositional function' we mean something which contains a variable $x$, and expresses a proposition as soon as a value is assigned to $x$. That is to say, it differs from a proposition solely by

\footnotetext{
${ }^{1}$ See also Macagno (2015), Macagno (2018), and Wyner (2016).
} 


\section{Goddu}

the fact that it is ambiguous: it contains a variable of which the value is unassigned. (Russell \& Whitehead 1925, p. 38).

Assuming there is a difference between the things that express propositions (presumably some sort of sentence in some language) and propositions themselves, then it is hard to make both sentences of what Russell and Whitehead say compatible, since the first makes them the sort of thing that express propositions, while the second makes them a kind of ambiguous proposition. Their gloss on ambiguity in terms of a variable with an unassigned value does not help. A variable is itself an expression and so the propositions themselves would not contain variables. The question is what does the variable express or represent? Presumably, some sort of hole or gap or incompleteness in a partial proposition.

I suspect one could go either way with a theory of propositional functions and so the ontology of argument schemes-schemes could be (i) sets of expressions with at least one variable or (ii) sets of incomplete propositions. In favor of propositions one might argue, firstly, that if one thinks it is possible for different expressions to express the same scheme, then since the expressions are different and the scheme the same, the scheme had better not be composed of expressions. For example, if one thinks that 'Anyone who respects Bob ought to respect George; $x$ respects Bob; hence $x$ ought to respect George' is just another way of expressing the same scheme as 'If $x$ respects Bob, then $x$ ought to respect George; $x$ respects Bob; so $x$ ought to respect George' then one will have to opt for propositional functions as incomplete propositions or propositions with 'holes.' We express these incomplete propositions via sentences with variables in them to represent the 'holes' and yet it appears that different such expressions can represent the same incomplete proposition. Secondly, if one takes seriously that argument schemes are arguments with 'holes' in them, and that arguments are composed of propositions, as many are inclined to do, then the 'holes' will be in the constituent propositions, and so propositional functions will be propositions with 'holes' in them.

Unfortunately, given (1), the second argument leads to incoherence. If we take seriously the claim that argument schemes are 
schemes with 'holes,' and take seriously the claim that logical forms, such as:

\section{S1: If $X$, then $Y ; X$; hence, $Y$}

are schemes, then the 'holes' may not merely be 'holes' in the constituent propositions, but 'holes' that encompass entire propositions. For example, the ' $X$ ' in $\mathrm{S} 1$ is not a proposition with a 'hole' in it, or an incomplete proposition, but at best a 'hole' that can be filled by entire propositions. We may be able to make the holes in a piece of swiss cheese bigger and bigger, but a hole that excludes all the cheese is not an instance of swiss cheese or even an incomplete piece of swiss cheese. The notion of an incomplete proposition that is just a 'hole' is incoherent, ${ }^{2}$ and yet logical forms require 'holes' that range over entire propositions. Hence, I opt here for propositional functions to be expressions with at least one variable, which could be just the variable itself. ${ }^{3}$

But what of the intuition that we can use different expressions to represent the same argument scheme as in the first argument above or that we could use different expressions to represent the same incomplete proposition as in the English, ' $x$ hates $y$ ' and the German, ' $x$ hasst $y$ '. Since they are in different languages, they are different expressions and yet they apparently express the same incomplete proposition.

Suppose we have a set of allowable substitutions for ' $x$ ' and ' $y$ ' in English and a set of allowable substitutions for ' $x$ ' and ' $y$ ' in German. Then, just as long as the set of propositions that are expressed from all the possible substitutions for ' $x$ ' and ' $y$ ' in ' $x$ hates $y$ ' in English is the same set of propositions as from ' $x$ hasst $y^{\prime}$ in German, then the two expressions pick out the same proposi-

\footnotetext{
${ }^{2}$ Some would argue that the notion of a proposition with parts, and so with one part missing, is incoherent (See, for example, Keller, 2013). But regardless of the metaphysics of propositions, the entirety of the proposition missing is not an incomplete proposition, so I need not take a stand on the metaphysics of propositions here.

${ }^{3}$ In an earlier presentation version of this work (Goddu, 2019) I, for ease of presentation, just stipulated that propositional functions were a sort of incomplete proposition. Subsequent reflection has led me to opt for expressions.
} 
tional function. ${ }^{4}$ In other words, propositional functions are equivalence classes of expressions.

We arrive then at the following definition of a propositional function:

D1: By a 'propositional function' we mean a class of expressions, each of which is either a variable or contains at least one variable, such that when each variable in each expression is replaced with an equivalent value, every member of the class expresses the same proposition.

As ungainly as this definition might at first appear, assuming that one (a) wants to take (1) seriously and (b) allow for the possibility of different expressions with variables ultimately picking out the same function, our options are limited. ${ }^{5}$

But if arguments are composed of propositions and argument schemes are composed of expressions, we lose the intuitive elegance of being able to say that argument schemes are schematic arguments because they are composed of schematic propositions. Perhaps the inelegance can be avoided by holding that arguments are themselves composed of expressions rather than propositions.

Of the three ontological candidates for arguments, viz, propositions, acts, or expressions, expressions is probably the least popular choice amongst theorists. One primary reason is that Anselm's Ontological argument in Latin, is still Anselm's Ontological Argument when translated into English or German. But since the

\footnotetext{
${ }^{4}$ I leave unanswered the question of whether the set of allowable substitutions for the variables in a propositional function is part of the function, in which case different substitution sets would entail different functions (and so the number of argument schemes would multiply accordingly) or whether one propositional function can be used with different domains for the variables.

${ }^{5}$ But not necessarily to just expressions. For example, one could take propositions to be sets of possible worlds and then take a propositional function to literally be a function such as $f$ ( $x$ is tall) that maps allowable substitution instances of $x$ to sets of possible worlds. Of course, we would still need to determine the nature of the allowable substitution instances for $x$-would those be expressions or the referent of those expressions? Also, we would still lose the notion that propositional functions were incomplete propositions since the notion of an incomplete set of possible worlds is incoherent.
} 
languages are different, the expressions are different and so if arguments are composed of expressions, we would have to say the Latin, English, and German versions of Anselm's argument are all different arguments.

But why not make the same move made above to explain how different expressions pick out the same propositional function, i.e., are in one particular equivalence class of expressions? Arguments, then, would be equivalence classes of expressions, i.e., all the expressions that express the same complex of propositions and Anselm's argument in Latin, or English, or German, would once again be one argument. There is however a significant difference between the case of arguments and the case of propositional functions. In the case of propositional functions, the choices were incomplete propositions or expressions. I chose expressions and then defined equivalence classes of expressions in terms of sets of propositions. Complete propositions were not one of the options for propositional functions. But in the case of arguments, propositions are one of the options for the components of arguments, so if one is ultimately relying on the propositions to define the equivalence class of expressions that are an argument, one should just say the propositions are the argument - after all, the propositions are doing all the work. Hence, despite the inelegance of arguments being composed of propositions and argument schemes (or schematic arguments) being composed of expressions, I still avoid the incoherence of incomplete propositions that are just 'holes' and account for the intuition that different expressions could express the same propositional function.

The incoherence of incomplete propositions that are just holes is a result of accommodating (1), that logical forms are schemes. But perhaps if we give up (1) we can avoid the incoherence. Perhaps. But the point of this paper is to try to articulate a theory of the nature of argument schemes that respects (1) and point out some of its consequences. Some of those consequences may be unpalatable enough for some to reject the resulting theory-I do not. Regardless, for now, I hold off evaluating the consequences of holding or rejecting (1), see especially section 4.2, until after the theory has been fully presented. I turn instead to a difficulty for T1. 


\subsection{A difficulty for $T 1$}

Suppose one takes seriously the claim that argument schemes are schematic arguments. ${ }^{6}$ Hence, every argument can be made into a scheme by schematizing at least one element of the expression of the argument, i.e., by replacing at least one element of the expression with a variable. So, consider the slightly stilted argument expression:

A1: Harry hates Billy. Hence, not possible that Harry impartially evaluates Billy's work.

Now consider some potential single variable schematizations of A1:

S2: $X$ hates Billy. Hence, not possible that $X$ impartially evaluates Billy's work.

S3: Harry hates $X$. Hence, not possible that Harry impartially evaluates $X$ 's work.

S4: Harry $X s$ Billy. Hence, Harry cannot impartially evaluate Billy's work.

S5: $X$. Hence, not possible that Harry impartially evaluates Billy's work.

S6: Harry hates Billy. Hence, $X$ possible that Harry impartially evaluates Billy's work.

S7: Harry hates Billy. Hence, not $X$ that Harry impartially evaluates Billy's work.

S8: Harry hates Billy. Hence, not possible that Harry $X$ evaluates Billy's work.

S9: Harry hates Billy. Hence, not possible that Harry impartially $X s$ Billy's work.

S10: Harry hates Billy. Hence, not possible that Harry impartially evaluates Billy's $X$.

S11: Harry hates Billy. Hence, not possible that $X$

\footnotetext{
${ }^{6}$ This sort of claim motivates what David Hitchcock calls bottom-up generation of schemes (Hitchcock, 2017, p. 226).
} 
S12: Harry hates Billy. Hence, not $X$.

S13: Harry hates Billy. Hence, $X$.

Depending on what can and cannot be properly replaced with a variable, a question I am not going to try to answer here, there may be even more single variable schematizations of $A 1$, such as:

S14: Harry $X$. Hence, not possible that Harry impartially evaluates Billy's work,

S15: Harry hates Billy. Hence, not possible that Harry $X$ Billy's work,

S16: Harry hates Billy. Hence, not possible that Harry $X$.

The maximal schematization is the eight variable:

S17: $X Y s Z$. Hence, $A B(X C D s Z$ 's $E)$.

There are lots of schematizations in between, such as the two variable:

S18: $X$. Hence, $Y$

or

S19: Harry hates Billy. Hence, not $X$ that Harry impartially Ys Billy's work,

or the three variable:

S20: $X Z s Y$. Hence, not possible that $X$ impartially evaluates $Y$ 's work.

All of these are schematic instances of Argument 1. But given $\mathrm{T} 1$, not all of them are argument schemes, since $\mathrm{T} 1$ requires that every sentence element of a target argument expression be turned into a propositional function. But this is true of only two of the fifteen given potential single variable schematizations, since only 
two elements are common to both premise and conclusion, viz 'Harry' and 'Billy.'

If all it takes to be an argumentation scheme is to be the schematization of some argument expression, then S4-S13 are schemes just as much as S2 and S3. Indeed, I suspect it is an interesting question what can and cannot be substituted in for $X$ in Harry $X s$ Billy in S4, such that it is true that it is not possible for Harry to impartially evaluate Billy's work. Of course, one might argue that Harry and Billy are just placeholders here and the real scheme of interest is S20, which does satisfy T1. But consider:

S21: Mephistopheles Xs God. Hence, not possible for Mephistopheles to impartially consider God's judgments.

In this case, given how unique Mephistopheles and God are supposed to be, we may really be interested in what values of $X$ would make the conclusion true for those two individuals. In other words, I am not sure we should rule out by definition that schemes cannot have complete proposition expressions as parts.

In addition, I also suspect that our maximal schematization, $\mathrm{S} 17$, is unlikely to be of much interest, since it will have too many disparate and unrelated instances to make it of any value, and yet it also satisfies T1. So, T1 as it stands appears to exclude certain items by definitional fiat and merely appealing to some sort of 'suitably general' principle will not explain why the maximal schematization is of limited to no interest. Hence, to avoid the definitional exclusion of many of the possible schematizations of A1, I also offer the following even more liberal definition of argumentation schemes:

T2: An argument scheme is a set of a set of proposition expressions or propositional functions and another proposition expression or propositional function, with at least one element being a propositional function.

Regardless of whether one adopts T2 (or accepts T1 and the concurrent challenge of explaining why S4-S13 and perhaps S21 should be excluded from the realm of schemes), either theory 
captures all existing cases of argumentation schemes. Any logical form such as:

S22: $X$ or $Y$; not $X$; hence, $Y$.

or

S23: If $X$, then $Y$; If $Y$, then $Z$; Hence, if $X$, then $Z$,

can be captured by T1, and anything that satisfies $\mathrm{T} 1$ also satisfies T2. Similarly, typical argumentation schemes such as:

S24: Source $E$ is an expert in subject domain $S$ containing proposition $A ; E$ asserts that proposition $A$ is true (false); Hence, $A$ is true (false.), (Walton, Reed, and Macagno, 2008, p. 310)

or

S25: Generally, case $\mathrm{C} 1$ is similar to Case $\mathrm{C} 2 ; A$ is true (false) in case $\mathrm{C} 1$; Hence, $A$ is true (false) in case $\mathrm{C} 2$. (Walton, Reed, and Macagno, 2008, p. 315)

satisfy both $\mathrm{T} 1$ and $\mathrm{T} 2$. In fact, all the schemes one can find in the literature satisfy both $\mathrm{T} 1$ and $\mathrm{T} 2 .^{7}$ Of course, recall we have the scheme:

\section{S18: $X$, Hence $Y$,}

which captures all possible simple arguments given that $X$ could be any proposition whatsoever (including a perhaps infinite conjunction) and $Y$ could be any proposition whatsoever.

\footnotetext{
${ }^{7}$ What if there are arguments with multiple conclusions? Then T1 and T2 are just definitions of simple argument schemes and then sets of schemes would be needed to capture complex schemes.
} 


\subsection{Critical questions?}

One might object that $\mathrm{T} 1$ and $\mathrm{T} 2$ do not capture all given argumentation schemes on the grounds that in many cases, schemes, especially defeasible schemes, are presented along with a series of critical questions and $\mathrm{T} 1$ and $\mathrm{T} 2$ include no mention of critical questions at all. Are critical questions part of schemes or not?

One standard answer is "no." Schemes have corresponding questions, but the questions are not part of the schemes themselves, but rather provide guidance for the proper evaluation of instances of the schemes. For example, Walton, Reed, and Macagno write: "The two elements together, the argumentation scheme and the matching critical questions, are used to evaluate a given argument in a particular case ...” (2008, p. 11). David Hitchcock writes:

As an aid to evaluation of the inference in an argument fitting a certain scheme, the theorist will provide a list of so-called "critical questions" to be asked corresponding to the conditions under which arguments of the scheme in question have a good inference (2017, p. 226).

Ian Dove and E. Michael Nussbaum (2018), citing the challenges of getting students to even recognize and utilize schemes, propose a strategy for argument assessment that generalizes the critical questions so as to be completely independent of any particular argument scheme.

Indeed, examination of the critical questions should lead one to believe that they are not constitutive of the scheme since the critical questions are not setting out conditions for an instance of a particular scheme being an instance of the scheme. For example, consider the question, "Are there other events that would more reliably account for the sign?", in a typical argument from sign. The answer is either "yes" or "no." But neither answer entails that the target argument is or is not an instance of an argument from sign. Rather those answers help an evaluator of a particular instance of an argument from sign judge whether the argument is a good one or not. In other words, the critical questions are not setting conditions for something to be an instance of the scheme in 
question, but rather setting conditions for something to be a good or acceptable instance of the scheme. We should keep identity conditions separate from goodness conditions, and so critical questions should not play a role in defining the scheme.

But despite the quotation given above, Walton, Reed, and Macagno also write: "The critical questions form a vital part of the definition of a scheme..." and "the complete set of linked premises employed in a scheme is thus the union of those given as premises and (the propositional content of) those listed as critical questions" (2008, p. 17). They provide an example in which critical question content gets added as explicit premises with the scheme 'appeal to expert opinion' in order to generate more and more refined and complex versions of the scheme.

Suppose we have some scheme $\mathrm{S}$ with associated critical questions, CQs. There is no doubt that we can incorporate the content of the CQs into $\mathrm{S}$ to create a new scheme S'. For example, suppose $S$ is " $X$ and $X$ is a sign of $Y$, so $Y$ " with CQs, "What is the strength of the correlation of the sign with the event signified?" and "Are there other events that would more reliably account for the sign?" Hence, $S$ ' might be " $X$ and $X$ is a sign of $Y$ and no other events more reliably account for $X$ and the correlations of $X$ with $Y$ in the context is sufficient, so $Y$."

Does S' have its own associated critical questions or not? If not, then there are schemes with no associated critical questions and so critical questions are not necessary for the identity of schemes. Indeed, in such a case, S' satisfies both $\mathrm{T} 1$ and $\mathrm{T} 2$ as does $\mathrm{S}$ without the corresponding CQs. But the objection to T1 and T2 on offer was that it was failing to account for the CQs, so the only way for the objection to continue is to claim that $S$ really is not a scheme after all, and only $S^{\prime}$ is, even though it has no CQs either and that seems arbitrary at best. We already know that we can take an argument ' $X$, so $Y$ ' and transform it into the argument ' $X$, if $X$, then $Y$, so $Y$ ' such that both arguments work or fall together. All that changes is the evaluation - if the inference of the original argument fails, then the conditional premise of the second argument is false and vice-versa. If the inference from $X$ to $Y$ is good, then the conditional premise of the second argument is true, and we are right back to determining whether or not $X$ is in fact true or 
acceptable or whatever the premise adequacy standard is that is in play. But incorporating the CQs of $S$ to generate $S$ ' is the exact same sort of transformation-if we compare instances of $S$ with S', what used to be questions about the premise adequacy or inference adequacy of $\mathrm{S}$ will now be questions about premise adequacy of S' for the newly added premises. For example, is it true that the correlation between $X$ and $Y$ is sufficient in the context or not? But just as no one denies that both ' $X$, so $Y$ ' and ' $X$, if $X$, then $Y$, so $Y$ ' are both arguments, no one should deny that both $\mathrm{S}$ and $\mathrm{S}$ ' are both schemes.

But suppose S' does have its own critical questions. Then the associated critical questions for $S^{\prime}$ can be incorporated as missing premises to create scheme $S^{\prime \prime}$ and we have our two options again for S' '. If it does not have CQs, then S', will satisfy T1 and T2 just as well as S and S' stripped of their CQs and saying $S^{\prime \prime}$ is the real scheme whereas $S$ and $S$ ' are not will again be arbitrary. If $S$ ', does have CQs, then once again we can raise the dilemma by creating S", Perhaps one might argue that eventually we will hit generic critical questions such as - are the premises adequate in the context, is the argument question begging in the context, do the premises sufficiently support (either by themselves or despite proximate potential counterexamples) the conclusion in the context? But since these questions can be asked of any argument, they are not doing any scheme individuating work-but that means the scheme is being individuated solely by its content independently of the generic critical questions, i.e., we are in the exact same situation we were in when we supposed we could have schemes without critical questions, i.e., we have a theory of schemes that satisfies T1 and T2. Hence, either we can have schemes without critical questions (which satisfy $\mathrm{T} 1$ and $\mathrm{T} 2$ ) or attempts to incorporate critical questions into schemes while also appealing to critical questions as part of the identity conditions of schemes generates an infinite regress.

\section{The proposed desiderata of schemes}

Both T1 and T2 are quite simple theories of the nature of argument schemes. I also suspect many theorists will not find them very 
satisfying since the resulting schemes will fulfill few if any of the roles traditionally asked of schemes. For example, Macagno writes of schemes that:

they allow classifying arguments in general categories. They bring to light the implicit rule of inference from the explicit premise(s) to the conclusion in an enthymeme, ...they guide the evaluation of arguments through the set of critical questions. On the other hand, they can be used for producing arguments. Considering the type of conclusion and the available evidence, the user can select the most appropriate scheme and make explicit the premises providing more support to his claim. (Macagno, 2018, p. 560.)

Hitchcock, citing Garssen (2001, p. 81), also mentions inventing and evaluating arguments, but also adds "describing how a certain group of people reason and argue" (2017, p. 229). So the proposed roles are (1) providing an argument taxonomy, (2) argument evaluation, (3) enthymeme reconstruction, (4) argument invention, and (5) describing sub-groups' reasoning patterns. I will go through each of these in turn.

\subsection{Argument taxonomy}

If schemes are types of arguments, then arguments that instantiate a particular scheme would be arguments of that type. Then, if we had a practically manageable table of argument types, we might end up with a useful taxonomy of arguments. Just as it is useful to be able to classify substances via the periodic table or living things via Linnaean taxonomy, so as to make predictions about how substances or living things will behave given their varying properties in varying circumstances, presumably it is useful to know what type of argument is being instantiated in a given circumstance. Godden and Walton, for example, write: "from a purely theoretical point of view, we agree with Garssen (1994, pp. 106107) that a minimal set of exhaustive, mutually exclusive schemes is desirable" (2007, p. 270). In addition, the hope expressed at the panel session on argument taxonomy at the second European Conference on Argumentation was that we get a complete but manageable set of schemes (see Hoppman 2018, Kienpointner 
2018, Macagno 2018, Wagemans 2018; but also Macagno, 2015, p. 184 and Hitchcock 2017, p. 236).

$\mathrm{T} 1$ and T2 may be complete, but the resulting list of schemes is not manageable. Firstly, there are an infinite number of schemes (since there are an infinite number of logical forms and an infinite number of propositional functions). But an infinity of taxonomical categories is generally not practically manageable. ${ }^{8}$ More significantly, the schemes accommodated by $\mathrm{T} 1$ or $\mathrm{T} 2$ are not themselves ordered in the way that, say, elements on the periodic table are ordered. So while, in principle at least, we could have an infinite number of elements ordered in terms of numbers of protons and neutrons in the atom, T1 and T2, by themselves do not imply any ordering of the resulting infinity of schemes. Additionally, though an infinity of elements is theoretically possible, what makes the periodic table useful is that the actually existing elements are finite and then grouped into eighteen groupings that share relevant properties and which then allows the desired predictions about behaviors in given circumstances. T1 and T2 both quickly generate an infinite number of actual arguments schemes and simultaneously provide no ordering or subgroupings of those schemes. Hence, the taxonomy generated by $\mathrm{T} 1$ and $\mathrm{T} 2$ will not be very helpful.

In addition, $\mathrm{T} 1$ and $\mathrm{T} 2$ may be exhaustive, but the resulting schemes are not exclusive, at least in the sense that any given argument is an instance of exactly one scheme. Arguments instantiate lots of different schemes. Just consider A1 and the very partial set of schemes I provided above. So, what type of argument is A1 exactly according to schemes? Note also that A1, along with all single conclusion arguments instantiates $\mathrm{S} 18: X$, hence, $Y$, so all arguments are of the same type? But if arguments instantiate multiple schemes and all arguments also instantiate one particular scheme, once again the taxonomy of $\mathrm{T} 1$ and $\mathrm{T} 2$ will not be very helpful.

\footnotetext{
${ }^{8}$ Hitchcock worries about 'top-down' methods of generation that 'risk a combinatorial explosion of unrealized abstract possibilities' (2017, p. 236). But given an infinite number of potential actual arguments each of which can be schematized in multiple ways, we will have an infinitude of schemes generated bottom up as well.
} 
Perhaps the solution is to resort not to argument schemes, but to types of argument schemes. For example, Walton, Reed and Macagno give 12 versions of an argument from popular opinion scheme (2008, pp. 311-313). Given T1 and T2, there will be many, many more schemes that count as examples of argument from popular opinion. Regardless, perhaps all the schemes of T1 and T2 could be categorized into a manageable group of scheme types, such that all the argument from popular opinion schemes were in one category and all the argument from expert opinion schemes were in another and all the disjunctive syllogisms ${ }^{9}$ in another, and so on.

This of course introduces the problem of how to type argument schemes, which I am not sure is any easier than the problem of typing arguments that the appeal to schemes was supposed to solve. But even if we suppose the problem is solvable, there is no guarantee that the typing of schemes is a consequence of schemes themselves. Indeed, much of the recent work attempting to classify arguments is in fact independent of the traditionally listed schemes such as argument from expert opinion, argument from sign, etc. The principles appealed to include such things as type of warrant involved (Blair 2000; Katsav and Reed 2004), semantic possibilities (Kienpointner 2018), purpose of argument/means of achieving purpose (Macagno 2015, 2018), type of proposition, i.e., value, policy, or fact (Wagemans 2018), etc. But these principles undergirding various attempts to classify arguments are not gleaned from schemes. These principles could be, and often are, applied directly to arguments to classify them into types. Many of these taxonomy projects could proceed without needing to talk about logical forms or arguments from pity or expertise at all. The result is a taxonomy of arguments for which the traditional schemes are, at best, instances of the categories generated by the principles rather than aids in developing the taxonomy. Indeed, often the resulting challenge, met with limited success, is to take the traditional categories such as 'argument from pity' or 'argument by appeal to expert opinion' or 'hypothetical syllogism' or whatever

\footnotetext{
${ }^{9} A l l$ the disjunctive syllogisms? (1) $A$ v $B, \sim A$, so $B$; (2) $A$ v $B, \sim B$, so $A$; (3) $A$ v $B$ v $C, \sim B, \sim C$, so $A$, etc.
} 
and make them fit into the categories that these various principles independently generate.

Perhaps a principle more closely tied with schemes could serve as the basis for the taxonomy. Hitchcock, for example, hints that "it makes sense to group all appeals to eye-witness testimony as belonging to a single scheme, since the same set of critical questions apply to all such appeals. The same is true of appeals to expert opinion" (2017, p. 297). Here Hitchcock is discussing using the sameness of critical questions to make different argument instances all fall under one scheme, but one might apply a similar method to make a variety of schemes all fall under one type. In other words, while, according to $\mathrm{T} 1$ or $\mathrm{T} 2$ there will be many 'appeal to expert opinion' argument schemes, they will all be appeals to expert opinion because they all share the same set of critical questions and that set will not be shared by other types of schemes.

For this suggestion to work as a viable taxonomy, types of argument schemes would have to correspond with particular sets of critical questions. But no such correspondence is forthcoming. Firstly, recall that logical forms are a type of scheme. Disjunctive syllogisms are distinct from hypothetical syllogism which are both distinct from affirming the consequent, and yet typically logical forms do not have distinct sets of critical questions associated with them, other than the generic are the premises adequate and is the connection between premises and conclusion adequate. Hence, some other principle, beyond appeal to critical questions would be needed to deal with all the possible logical forms. Secondly, consider the following schemes:

S26: If $X$ is an authority about $Y$, and says $Z$ within the domain of $Y$, then $Z$ is most likely to be true. $X$ is an authority about $Y$ and says $Z$ within the domain of $Y$. Hence, $Z$ is most likely to be true.

S27: $X$ is an authority about $Y$ and says $Z$ within the domain of $Y . X$ is an authority in domain $Y$ because $X$ has property $A$, $B$, and $C$. $W$ also has properties $A, B$, and $C$, but $W$ says $\sim Z$. Hence, we should withhold judgement about $Z$. 
S26 appears to be both an argument by authority and a modus ponens. S27 appears to be an argument by authority and an argument by analogy and a balance of considerations. But since there are also arguments that are modus ponens that are not arguments by authority as well as arguments by analogy that are neither balance of considerations nor arguments by authority, no set of critical questions is going to both demarcate S26 and S27 as arguments from authority while also distinguishing them from modus ponens or argument by analogy, etc. ${ }^{10}$ Finally, actual attempts at computer modelling of schemes usually involves making the schemes fit a defeasible modus ponens form. (Walton, Reed, and Macagno, p. 364.) But if defeasible modus ponens is its own kind of scheme and all other schemes can be reduced to it, then no set of critical questions will both distinguish defeasible modus ponens from other types and yet allow all other types of schemes to be reducible to defeasible modus ponens.

I suspect that these worries generalize to almost any attempt to provide a neat and manageable taxonomy of arguments, let alone argument schemes - there are just too many ways of arguing that overlap with each other to get a complete and workable taxonomy. For example, if one attempts to ameliorate the overlap problem by appeal to some sort of hierarchy of schemes, one would still need to account for all the possible ways of schematizing A1-Harry hates Billy. Hence, not possible that Harry impartially evaluates Billy's work - that actually provides and answer to the questionwhat type of argument is A1? All the single variable schematizations provided, S2-S17, certainly do not appear to neatly fall under one more general type. After all, S17-XYs Z. Hence, $A B(X C$ $D s Z$ 's $E$ ) and S18- $X$. Hence, $Y$ are both presumably top node schematizations of A1 and yet are also, presumably, not of the same type. But then there is no neat hierarchical tree of schematizations that would allow us to type A1, but rather than overlapping

${ }^{10}$ Walton, Reed, and Macagno note this problem with existent categorization methods, including their own. For example, the write: "The slippery slope argument is often such a complex argument, combining many other subarguments of different types, that it is impossible to classify it under any single heading" (p. 351). 
web of schematizations that would not allow a coherent or useful typing of A1.

Given T1 and T2, there are too many schemes with too much overlap for a manageable taxonomy of schemes to be forthcoming. I conclude this section with Hitchcock's own recent re-evaluation of work on schemes (including his own):

Argument and reasoning are too varied for such neat pigeonholing. Indeed, in using any taxonomy of schemes as a device for analyzing and evaluating arguments, one is at serious risk of distorting the discourse or text to fit into one's taxonomy (2017, p. 297). ${ }^{11}$

\subsection{Argument evaluation}

Schemes aid evaluation of arguments via the set of associated critical questions. T1 and T2 do not have critical questions as part of the scheme, so the schemes themselves will not be of any help in this regard. Of course, neither theory prohibits schemes from having associated questions that may aid in the evaluation of instances of the scheme - the critical questions, however, remain separate from the identity of the scheme.

In section 2.3 above, I examined one attempt to incorporate critical questions into the identity of the scheme by incorporating the content of the questions as additional premises. That attempt generates either regress or arbitrary exclusion, but there is another way to make critical questions a part of the scheme that avoids these problems. Consider, for example:

T3: An argument scheme is a set of (i) a set of proposition expressions or propositional functions, (ii) another proposition expression or propositional function, with at least one element of (i) or (ii) being a propositional function, and (iii) a set of critical questions.

T3 doesn't try to make the elements of (iii) into elements of (i) and so avoids regress.

\footnotetext{
${ }^{11}$ See also Dove and Nussbaum, 2018, p. 262
} 
I am not denying that one could adopt T3 and so, by definition, make schemes relevant to argument evaluation. The question remains, however, whether we ought to adopt T3 over either T1 or T2. I suggest 'no.' Firstly, if anything that satisfies T3 counts as a scheme, then $\mathrm{T} 3$ radically increases the combinatorial options for different schemes, and so only exacerbates the problem of schemes satisfying the other desiderata of a theory of schemes. Secondly, theorists generally prefer to keep criteria of identity and criteria of evaluation separate, so why, in this instance, make criteria of evaluation part of the definition of what a scheme is? Thirdly, the nature and utility of critical questions are themselves a source of controversy in the literature, so why incorporate this extra controversy into the very definition of a scheme? For example, one criticism of the appeal to critical questions is that they add nothing over and above our standard criteria for evaluating arguments. For example, as Hitchcock (2017, p. 297) points out, it looks like we can reformulate all critical questions into single questions concerning premise adequacy, background assumptions, and exceptions, i.e., questions that apply to all arguments. But if the critical questions are just versions of questions that apply to all arguments, then including them as part of the identity conditions of schemes is pointless. Indeed, if (1) is true and logical forms are argument schemes, then the relevant critical questions appear to just be the generic, "are the instances of the premises true?" and "is the argument form valid?", which we do not typically include as part of the identity conditions of those forms.

David Godden and Douglas Walton (2007) concede that critical questions are grounded in general standards of argument adequacy (they focus on the typical R.S.A. standard), but go on to justify their use by claiming "there may be typical, or common ways in which the R.S.A. cogency conditions could apply to arguments of a given schematic type that would not typically apply to other common types of argument" (p. 279). In other words, a critical question might focus the evaluator's attention on the more typical ways a particular premise such as " $A$ is an expert in domain $D$ " might fail to be acceptable or sufficient, whereas the generic "is the premise acceptable" does not. Granted. But such a response at best justifies that we might find it useful to appeal to associated 
critical questions (for at least some schemes) rather than just generic argument adequacy conditions. It does not justify that we ought to make critical questions part of the identity of schemes themselves. An advocate of T1 or T2 who accepts that at least some schemes have associated critical questions, can just as easily appeal to Godden and Walton's (see also Blair, 1999, p. 56) argument to justify why it is useful for some of the schemes to have associated questions as an advocate of T3 can.

If critical questions are to be essential to scheme identity, then at least some of the critical questions must be something other than specific forms of generic adequacy conditions. But I see no evidence that any of the critical questions are anything other than specific forms of generic argument adequacy conditions. Hence, I see no reason to make critical questions part of the identity of particular schemes as in T3. Critical questions may be relevant to the evaluation of at least some arguments, but schemes by themselves, given $\mathrm{T} 1$ or $\mathrm{T} 2$, are not.

\subsection{Enthymeme reconstruction}

If we had a set of schemes against which to compare argument texts, then we could recognize incomplete argument texts and use the schemes to fill in the missing pieces. For example, in response to an objection by Robert Pinto (2003) that schemes will not help with argument reconstruction since we will have to presuppose the missing elements to identify the partial argument text as an instance of a scheme rather than use the scheme to identify the missing elements, Godden and Walton write:

The role of a scheme at this stage of analysis is not to supply missing material to an argument, but to serve as a model for comparison. It is by comparing the argumentative material presented in a case with the known schemes that an argument can be classified as an instance of a certain scheme (2007, p. 272).

And presumably, once one has successfully typed the argument via a given scheme, one is in a position to add the relevant missing material. 
Ignore for the moment skepticism (see Hitchcock 1998 or Goddu 2016) concerning enthymemes and ignore for the moment that Walton, Reed, and Macagno (2008. pp. 207-210) even note some other possibilities for dealing with enthymemes such as Peircean abduction or appeals to background/common knowledge. Even if we suppose that there are cases in which we want to reconstruct arguments from texts such that some components of the final argument are not explicitly expressed in the text, schemes, at least as conceived here, will not play any relevant role in typing the argument and subsequently determining the missing material to add. Why? Because for any given, allegedly incomplete, argument text, there will be countless distinct schemes for comparison that match the given material (including some that exactly match the given material except for the presence of at least one variable) and yet have quite diverse additional material as part of the scheme. Hence, the mere fact that the presented argumentative material matches a particular scheme is not enough to say the argument is of that scheme type, since there will be myriad other distinct schemes that match the presented argumentative material as well.

Even if, contra Pinto, one thought appeal to schemes could somehow directly indicate the missing material to add, T1 and T2 will not do this job either. For any proposed extra premises, there is a scheme that contains the original text (perhaps with some elements made into variables) and expressions of the added premises (again with some elements perhaps made into variables.) Hence, the existence of a given scheme is not determining which premise should be added, since so many schemes exist, that there are schemes for any possible added set of extra premises. Even if we restrict ourselves to 'plausible' candidate schemes such as adding some sort of conditional, or a generalization of some sort, or a definition, or some sort of typical nature claim or ... there will be a scheme for each of the plausible candidates. Assume for the moment that, at least in some cases, some of the plausible candidates are wrong, while at least one is correct. But whatever it is that is making the one particular candidate correct in that situation it is not the fact that there is a scheme according to which that candidate fits with the explicitly given text, since that is true for all 
the plausible candidates. Hence, given T1 or T2, something other than the appeal to a scheme is what should guide determining argument type or determining which missing components to add.

\subsection{Argument generation}

There is a trivial sense in which schemes aid in the generation of arguments. Take any instance of a scheme according to T1 or T2. Substitute in allowable terms for the variables, and voila, an argument. If this is all that is meant by schemes aiding in argument generation or invention, then $\mathrm{T} 1$ and $\mathrm{T} 2$ do satisfy this desideratum. But I suspect argumentation scheme theorists have something more robust in mind. For example, Macagno writes: "Considering the type of conclusion and the available evidence, the user can select the most appropriate scheme and make explicit the premises providing more support to his claim" (2018, p. 560).

On its face, this just seems like a version of the enthymeme reconstruction problem, except the argument author (as opposed to the interpreter) has only bits and pieces - a conclusion and available evidence and wants to know what else needs to be included (presumably to increase the likelihood of having a successful argument). But again, appeal to schemes will not help here (even if we exclude schemes that we know in advance are unlikely to meet the sufficient support standard) since there will be too many schemes available to choose from. In addition, being able to appeal to the type of conclusion or available evidence to pick out an appropriate scheme presupposes that schemes correlate with the type of conclusion or available evidence and that is not true of all schemes. It is certainly not true of logical forms - which are independent of content (except for the 'logical connectives'). Finally, being able to determine the scheme based on the type of conclusion or available evidence, presupposes we have a manageable argument or argument scheme taxonomy, both of which we have already seen are problematic, given $\mathrm{T} 1$ or $\mathrm{T} 2$. 


\subsection{Describing sub-group's reasoning patterns}

Plenty of schemes will do this for any given sub-group and its reasoning. Indeed, too many will do this to make the schemes theoretically useful. Since any arguing will instantiate multiple schemes, we can describe the very same reasoning in many ways, and so if there is something useful to be said about the arguing instantiating one of the myriad schemes rather than others, the schemes themselves will not be the determining factor.

This isn't to say that there is nothing useful, in certain disciplines, from recognizing that certain groups use a fairly common pattern of reasoning and giving a name to that pattern and using that name to aid in communication to other scholars. Adhering to $\mathrm{T} 1$ or $\mathrm{T} 2$ in no way prohibits theorists from doing this sort of thing. ${ }^{12}$

\subsection{A radical consequence}

The general reason T1 and T2 fail to satisfy many of these desiderata, at least simultaneously, is that there are too many schemes and single arguments satisfy too many of the schemes (indeed recall all single conclusion argument satisfy S19, X, so Y) for schemes to be what is doing the desired work. Assuming there are no other unrecognized benefits accruing from $\mathrm{T} 1$ or $\mathrm{T} 2$, then $\mathrm{T} 1$ and T2, as a theory of the nature of argument schemes would have something like the following as a consequence:

GT1: Argument schemes are not a theoretically significant part of a general theory of arguments and argumentation.

Just as the number of premises an argument has is a piece of data we can provide about an argument, so too are the possible schematizations of that argument. But just as the number of premises of an argument is theoretically irrelevant, so to, according to GT1, is the set of schemes that a given argument instantiates.

\footnotetext{
${ }^{12}$ For example, Jean Goodwin has told me in conversation that this sort of thing is quite common in rhetoric/communication studies, but also that there is much less concern about constructing a theory of such patterns that is complete and manageable.
} 
GT1 is very simple - argumentation schemes are theoretically irrelevant. Given the amount of work that has been done on argumentation schemes I expect this consequence to be extremely unpalatable. I turn next, then, to considering ways to avoid this radical consequence.

\section{A reductio?}

Given that T1 and T2 fail to satisfy the standard desiderata of a theory of argument schemes, and as a likely consequence, argument schemes have no significant theoretical role to play in argumentation theory, one might take this as a reductio of $\mathrm{T} 1$ and $\mathrm{T} 2$. If they do not satisfy the primary roles desired of argument schemes, then they are not adequate theories of the nature of argumentation schemes despite their simplicity. This, of course leaves the challenge of determining what argumentations schemes are and none of the existing takes on argument schemes are particularly good at justifying that they in fact satisfy these roles either. At best, they offer promissory notes that we can achieve a notion of schemes that provides a complete, manageable list of schemes that will satisfy at least a significant proportion of the desired outcomes. I remain skeptical that we will be able to simultaneously get 'complete' and 'manageable', let alone also satisfying the desired outcomes. While a full defense of my skepticism would require another full paper, I turn now to two strategies one might adopt to try to avoid the negative consequences adduced so far.

\subsection{Restricting the class of schemes?}

One straightforward solution is to limit what counts as a scheme. For example, as we saw in the beginning, Walton, Reed, and Macagno talk about schemes as "structures of common types of arguments". Walton and Godden define schemes as: "stereotypical patterns of defeasible reasoning that typically occur in common, everyday arguments" (Walton and Godden, 2007, p. 267). So perhaps schemes are not schematic versions of any possible argument, but merely the forms of 'common' or 'stereotypical' types of arguments used in everyday discourse. Hence, (1) is false, since only the logical forms that are commonly instantiated are schemes, 
along with the typical fallacies and other common argument structures such as argument from authority, expert opinion, etc. Presumably, the hope would be that this restricted class of entities would be much more manageable than the class set out by either $\mathrm{T} 1$ or $\mathrm{T} 2$.

Firstly, even if this class of entities, once concretely articulated, was much more manageable, I doubt that it is theoretically interesting. For example, a scheme such as:

S28: God commands that we do $x$, so we ought to do $x$,

may have had many more instances than it has now. But the mere fact that what was once a common way of arguing is now no longer a common way, or at least a significantly less common way, of arguing, or vice versa, should have no bearing on what arguments or argument schemes are as theoretical concepts we use to explain the phenomenon of argumentation through time. Hydrogen and helium make up $98 \%$ of the universe. Oxygen and nitrogen make up $99 \%$ of the earth's atmosphere. Hydrogen and oxygen make up $96.5 \%$ of earth's oceans. Oxygen, silicon, aluminum, iron, calcium, sodium, potassium, and magnesium make up $98 \%$ of the earth's crust. Yet despite the fact that these elements are the most common elements, we certainly do not restrict our theory of the building blocks of the universe to just these elements, rather our theory extends to all the naturally occurring elements, the human created elements, and even the possible elements beyond those. Similarly, our theory of arguments and their structures and forms should not be limited to the common or stereotypical cases. After all, as theorists, we are interested in not merely explaining what commonly happens, but what does happen, what could have happened but didn't, what is unlikely to ever happen and why, and so on.

This is not to say that we should not focus the majority of our research attention on understanding the most common ways of arguing, any more than we should limit studying the properties and interactions of hydrogen, oxygen, and nitrogen. What I am suggesting is problematic is trying to make a theoretical category out of what is most common or stereotypical-our theories need to 
explain not merely what is common or stereotypical, but what is uncommon and why and what is merely possible and why and perhaps even what is impossible.

Secondly, even if we force our theory to have a category that is "the forms of common or stereotypical arguments" I still doubt the result will be manageable and theoretically useful simultaneously. On the one hand, I suspect there will still be an infinite number, or at least an unmanageable number, of potential schemes, especially if one considers the schemes common to a particular discipline or context. For example, consider the combinatorial explosion of such elements of reasoning as: ' $x$ struck $y$,' ' $x$ slapped $y$,' ' $x$ punched $y$,' ' $x$ spit at $y$,' ' $x$ bit $y$,' ' $x$ kicked $y$,' ' $x$ threw $x$ 's shoe at $y$,' etc., so ' $x$ ought to be punished,' ' $x$ ought to be sanctioned,' ' $x$ ought to be chastised,' ' $x$ ought to be shown a red card,' ' $x$ ought to be shown a yellow card,' ' $x$ has committed a foul,' etc., or even the more general, ' $x$ is an expert in $y$ ' and ' $x$ says $z$,' ' $x$ wonders whether $z$,' ' $x$ hypothesizes $z$,' ' $x$ suggests $z$ ' etc., so 'we ought to believe $z$,' 'we ought to suspect $z$,' we ought to hypothesize $z$,' etc. Add in all the common patterns of reasoning from specific scientific disciplines, specific games based on their specific rules, and all the variations on more general schemes like arguing from authority, pity, etc., and we will still have an unmanageable number of schemes.

We could try to solve this problem by not just restricting ourselves to common reasoning patterns, but general forms of these common reasoning patterns. For example, one might try to subsume all the specific cases of the first example given above under the more general scheme:

S29: $X$ made contact of type $Y$ with $Z$. Contact of type $Z$ in context $C$ deserves a response of type $R$. $X$ and $Z$ are in context $C$, so $X$ ought to receive response $R$.

While I suspect a full review of treating argument schemes as general forms of common reasoning patterns would require a separate paper in itself, I can here at least suggest some challenges facing such a proposal. 
Firstly, to actually solve the 'manageability' problem, one would have to deny that all the common reasoning patterns themselves are schemes. The only things that are schemes are the general forms. But now the theorist has the added challenge of providing a principled distinction between a mere reasoning pattern, and the general form of one. This challenge is especially pressing, given that modus ponens, say, is supposed to be itself a scheme and yet also looks itself to be a common reasoning pattern.

Secondly, to even have a chance of making the set of schemes manageable, these general schemes are going to have to be pretty general - but then the general forms are going to be quite divorced from actual commonplace cases of reasoning. For example, actual cases of reasoning are not themselves usually instances of S29. I justify the giving of a red card in the context of a soccer match by saying that Blue 29 punched Red 12. I do not say that Blue 29 made contact of type 'punch' with Red 12 and contact of type 'punch' in the context of a soccer match deserves a response of type 'red card' and Blue 29 and Red 12 are in the context of a soccer match, so Blue 29 ought to receive a red card. So, given that the actual instances of arguing rarely satisfy the explicit form of the generalized scheme, to actually tell what type of argument is in play one would not only need to be able to schematize the argument, but then generalize the resulting pattern as well in order to get at the actual scheme being instantiated. But, as we have already seen, there are many ways to schematize a given argument, so it is still possible that a given argument will instantiate numerous different general forms.

Thirdly, how general can the general form get? ${ }^{13}$ Every argument has the general form ' $X$, so $Y$,' but that form is not theoretically useful as a scheme. I suspect that to be useful, schemes have to have at least one 'argumentative constant' other than the illative, such as in:

S30: ' $X$ is an expert in $Z$, so $Y$.'

\footnotetext{
${ }^{13}$ See also Blair 2001 and Walton and Godden 2007 for more discussion of the generality issue.
} 
But I suspect the number of general forms with at least one 'argumentative constant' other than the illative is still extremely large (more on this in the next section). And if we need more than one 'argumentative constant,' then how many do we need and why?

Fourthly, do the general forms need to build in context as I have done in S29 or not as in S30? On the one hand, building in context makes the argument scheme appear more plausible since one can see the connection between $X$ 's action say and $X$ receiving response $R$, whereas in the case of $\mathrm{S} 30$, there is no common variable between the premise and the conclusion, which suggests there are going to be many extremely bad instances of S30. But on the other hand, theorists (a) want to be able to apply schemes across contexts and (b) want to be able to separate the argument being made from the context in which it is being made, and both of these items are more challenging, if not impossible, if context is built into the scheme. Note also that arguers usually do not explicitly appeal to the context in which they are arguing as part of their argument, so once again these general forms appealing to context would be divorced from most actual instances of reasoning.

Again, I do not take these suggestions to prove that no way of limiting the class of argument schemes allowed by T1 or T2 will work. But the challenges of providing such a theoretically significant and manageable set of argumentation schemes are daunting to say the least.

\subsection{Reject (1)?}

At several junctures above, we saw that holding (1)-logical forms are a type of argument scheme-was generating various problems for the argumentation scheme theorist, such as an explosion of the number of schemes and schemes with entire propositions as variables. So a straightforward solution might be to just reject (1). Indeed, in presentations of earlier versions of this work, the most common audience response was to propose giving up (1). ${ }^{14}$ The option considered above to try to restrict schemes to

\footnotetext{
${ }^{14}$ For example, at the Ninth Conference of the International Society for the Study of Argumentation, 2018.
} 
'common or stereotypical ways of arguing' is itself an instance of rejecting (1).

The primary purpose of this paper is to explore the consequences of a theory of the nature of schemes that does respect (1). If the consequences are unpalatable enough, one can certainly try to generate a theory that rejects (1) - but what such a theory would look like is not the primary concern of this paper. For those who wish to keep the desiderata of a theory of schemes outlined in section 3 and yet reject (1), I leave it as a challenge to actually produce such a theory.

To see the scope of this challenge I finish this section with the following observation about a theory of the nature of schemes that rejects (1).

Consider:

S31: All $X$ are $Y$ and some $X$ are $Z$, so some $Z$ are $Y$.

and

S32: $X$ said $Y$ in domain $Z$ and $X$ is an expert in domain $Z$, so it is highly likely that $Y$.

The challenge is to come up with a principled reason to reject S31 as a scheme, but keep S32, while at the same time respecting the desiderata of section 3. I admit that I am hard pressed to even imagine what a principled reason for keeping S32, but rejecting S31 is going to look like, let alone one that will also keep the desired outcomes of a theory of schemes. I strongly suspect that several of the arguments given in section 4.1, such as the likelihood of there still being an infinite number of schemes even without (1), will carry over to any attempt to reject (1). And if there are still an infinite number of schemes (or scheme types) even without holding (1), then many of the desired outcomes of a theory of schemes remain unachievable.

But perhaps one is willing to give up not just (1), but also the desire for a general and complete theory of schemes. In other words, to give up the quest for any GT with respect to argumenta- 
tion schemes. As one commentator on an earlier version of this work put it: "schemes are not an exhaustive classificatory system of arguments into their natural kinds. Rather they supply a somewhat arbitrary, ad-hoc typification of arguments into recognizable commonplaces or Aristotelean Topoi." 15

I have absolutely no quarrel with such a position since I strongly suspect that at best schemes, as commonly understood in the literature, are ad-hoc groupings of commonplace or stereotypical reasoning patterns (including some, but not all, logical forms). However, if one takes this route, then one should give up on trying to generate a complete and exhaustive taxonomy of all arguments, providing a mechanism for filling in any enthymeme or generating any argument whatsoever, or evaluating any sub-groups arguments on the basis of schemes. In other words, one would have to give up on using the schemes to achieve the desiderata outlined in section 3.

Note that I am not at all saying that there is no practical benefit from availing ourselves of such ad-hoc groupings. For example, individuals may be able to reason better or more efficiently or faster with at least a rudimentary grasp of some of these ad-hoc groupings or perhaps some could use the groupings as models in argument generation and so increase the chances of making a cogent argument. Navigating via the north star has practical benefits; using the rule of 72 to calculate doubling time ${ }^{16}$ has practical benefits, but the north star is not a fundamental entity in a theory of navigation or spatial position and the rule of 72 is not a fundamental part of theory of interest rates or number theory. Similarly, argument schemes on such a view would have no place in a fundamental theory of arguments. We should just not try to take the ad-hoc rough and ready groupings that may help us, say, identify a likely flaw in a particular bit of reasoning in some contexts, and try to generalize those groupings into a theory applicable to all arguments and argumentation.

\footnotetext{
${ }^{15}$ Unknown reviewer.

${ }^{16}$ For example, at $10 \%$ annual return, we would expect our money to double in roughly $72 / 10$ or 7.2 years.
} 
What then of a theory of schemes? I turn now to a modest proposal that may mitigate some of the negative and skeptical results achieved so far.

\section{A slight refocusing?}

$\mathrm{T} 1$ and T2 are both simple, elegant theories of the nature of argumentation schemes. Just as there are lots and lots of arguments there are lots and lots of schemes these arguments can instantiate. Unfortunately, the sheer number of schemes makes them unwieldy for achieving the theoretical benefits many have hoped to gain by appealing to schemes. Given that I currently see no plausible way of defining schemes to actually achieve the desired theoretical benefits, I choose simplicity and elegance. But I also suspect a slight refocusing might make us less worried about failing to achieve the theoretical desiderata.

What are we getting when we schematize (and generalize) reasoning? Presumably we are trying to understand the 'argumentative force' of whatever is being left as the 'argumentative constant' in the scheme (and how various things left as constants will interact with each other). A simple example-leaving 'or' and 'not' as constants in ' $P$ or $Q$ ', 'not $P$ ', will, given true premises, entail ' $Q$ '. In other words, we gain information about how 'or' and 'not' argumentatively interact. Similarly, leaving 'is an expert in' and 'testifies that' as constants in ' $X$ is an expert in $Y$ ' and ' $X$ testifies that $Z$ ' we can wonder what conditions need to hold in order for something plausible to be inferable from instances of ' $X$ is an expert in $Y$ ' and ' $X$ testifies that $Z$ ' working together. Of course, if we change either one of the 'constants,' we can change the results. For example, consider replacing 'testifies that' with 'asserts that,' or 'wonders whether,' or 'supposes that,' or 'hypothesizes that,' or 'suggests that,' etc.

So, the proposal then is to focus our attention on propositional functions ${ }^{17}$ rather than argument schemes. Instead of focusing our attention on trying to identify arguments from expert opinion say,

\footnotetext{
${ }^{17}$ Perhaps these propositional functions are the atoms that Hoppman (2018) is looking for, though as it stands there are going to be too many of these 'constants' for the sort of taxonomy Hoppman desires.
} 
we should focus on identifying appeals to expertise, i.e., instances of the propositional function, ' $X$ is an expert in $Z$.' Of course, given that, as argumentation theorists, we are presumably ultimately interested in distinguishing good arguments from bad arguments, we are likely to be very interested in determining the argumentative force of such a propositional function both across various contexts and in relation to other propositional functions such as ' $X$ says $Y$ ' or ' $X$ doubts that $Y$,' etc. Some combinations of propositional functions in certain contexts may be more likely to have good argumentative results, and so, unsurprisingly, we can expect more instances of those combinations to appear rather than the combinations less likely to achieve argumentative success. But to understand the 'argumentative force' of 'is an expert in' we need to understand not only the combinations of functions (at least one of which contains the 'logical' constant 'is an expert in') that do appear frequently, but understand why other combinations do not, and perhaps understand which combinations ought to appear more (or less) frequently given our desire not merely for argumentative success, but rationally persuasive arguments.

One could define classes of arguments in terms of these propositional functions. For example, an argument from expert opinion could be the class of arguments involving ' $X$ is an expert in $Z$ ' (or perhaps involving it essentially). Similarly, conditional arguments would be arguments involving (or involving essentially) the propositional function 'If $X$, then $Y$.' But I doubt the resulting classes would be very theoretically interesting. On the one hand, there would be plenty of overlap between classes - any argument involving ' $X$ is an expert in $Z$ ' will be transformable into a defeasible modus ponens form, so such transformed arguments will be both conditional arguments and expert opinion arguments. Also, such classes are likely to be extremely heterogenous groupings of arguments. For example, some conditional arguments will be good and some will be bad and some will be valid, i.e. those also involving ' $X$ ' getting us to ' $Y$ ' or 'not $Y$ ' getting us to 'not $X$ ', and some will not be generally valid, i.e. those also involving ' $Y$ ' getting us to ' $X$,' or 'not $X$ ' getting us to 'not $Y$,' or ' $Z$ ' getting us to whatever. Some will be indicative conditionals, some will be subjunctive conditionals, some will be counterfactuals, and so on. 
Hence, the class of conditional arguments will be too heterogenous to be theoretically significant.

Of course, some combinations of various propositional functions may appear enough in our argumentation that they get special names, such as modus ponens or argument from sign, etc. But to understand why those combinations may appear more than others we probably have to understand the argumentative force of the component propositional functions and how they interact with not just the other propositional functions in the combination under examination, but how they interact with other propositional functions that could have been used but were not. The mistake is to think that just because there are more prevalent combinations of propositional functions, there must be a theoretically useful underlying structure of types of arguments. Rather we should expect our desire to demarcate good from bad arguments in various contexts to itself generate clusters of combinations of functions that are more likely to be used (because they are good or successful or because they are easily confused with clusters that are good). But just as formal logic is interested in demarcating the good and bad interactions of such connectives as 'or,' 'not,' and 'if, then,' so too should we expect more general argumentation studies to be interested in demarcating the good and bad interactions of 'expertise,' and 'saying,' and perhaps a strong emotion such as 'hatred' in making inferences about what is said more or less reasonable. The advantage formal logic has is that, classically at least, the connectives are inter-definable, so it is easy to generate a finite set of valid forms that can be used to determine validity/invalidity for any target argument (at least relative to the chosen formal structure). The typical 'constants' of most defeasible schemes, however, are not inter-definable, and so, given the sheer number, a function of the combinatorial power of our languages, of such potential constants, we should not be surprised that we cannot generate a manageable set of defeasible forms from such functions, if at all.

To sum up: Specific focus on particular schemes, is perhaps better construed as attempts to understand the argumentative/inferential force of very particular 'argumentative constants' such as 'is an expert in' or 'has very strong emotion... towards...' or 'if ..., then ...' etc., through in-depth analyses of how those 
functions interact with other functions and the preconditions or scope of application of those functions for generating acceptable inferences. Refocusing on particular propositional functions will not generate any of the outcomes desired of schemes, such as providing a taxonomy of arguments or helping with the so-called problem of enthymemes, ${ }^{18}$ since the sheer number of possibilities involved in argumentation and the expressive power of our language likely precludes ever achieving such outcomes. At the same time, facility with (i) recognizing crucial functions, (ii) the interactions amongst propositional functions, (iii) the conditions needed for acceptable instances of functions in various contexts ${ }^{19}$ and (iv) the resulting inferential strengths and weaknesses, without trying to force every argument to fit into a theoretically dubious set of argument schemes, can be useful in the evaluation of arguments and the invention of one's own (hopefully stronger) arguments. More generally, focusing on propositional functions rather than schemes makes one more open to alternative combinations of such functions rather than trying to subsume every argument into a fixed set of schemes.

\section{Conclusion}

Despite significant and important analyses of many particular schemes, which I interpret as attempts to understand the argumentative force of such propositional functions as ' $X$ is an expert in $Z$ ' or ' $A$ is a sign of $B$ ' or 'Act $X$ has value $Y$, etc., there has been little success in generating an acceptable underlying theory of argumentation schemes. T1 or T2 are both simple and elegant theories of the nature of argument schemes that encompass all identified schemes in the literature and all logical forms and many more potential schemes besides.

One consequence of T1's or T2's power to capture all these schemes in a simple way, however, is a tremendous proliferation

\footnotetext{
${ }^{18} A$ or $B$, so $A$-what is the missing function? 'Not $B$ '. You sure? Why not 'If $B$, then $A$ ' or 'If $A$ or $B$, then $A$,' or 'The probability of $B$ is less than one in a trillion,' or...

${ }^{19}$ I suspect that at least some critical questions are trying to point toward adequacy conditions for acceptable uses of particular propositional functions.
} 
in the number of potential schemes. Given the sheer number of potential arguments we should not be surprised by the sheer number of schemes. Unfortunately, the number of schemes given by $\mathrm{T} 1$ or T2 precludes schemes from fulfilling any of the outcomes theorists have hoped to gain from schemes, such as providing a manageable taxonomy of arguments, aiding in enthymeme reconstruction, evaluating or generating arguments, or describing particular sub-groups' reasoning processes. Indeed, if no other potential theoretical benefits from T1 or T2 are forthcoming, an even more radical consequence is that argumentation schemes are generally theoretically irrelevant. To avoid that result, the onus is on defenders of argument schemes to provide an account of their nature that could be part of a general, non-arbitrary, theory of schemes and yet still gives them a theoretically significant role to play.

Failing that, should we then abandon T1 or T2? No. We should abandon the attempt to achieve the desired outcomes, if they really are desired, via a theory of schemes. Perhaps some of those outcomes can be achieved via other means. For example, nothing I have said here precludes the possibility of appealing to various scheme independent principles to try to generate a taxonomy of arguments - whether any such taxonomy would be both manageable and theoretically significant remains to be seen.

What has the long standing attempt to generalize and schematize arguments gained us? A much better understanding of the argumentative force of various 'constants' such as 'is a sign of,' 'is an expert in,' 'or,' and so on, both in various combinations and in interaction with each other. Hence, I recommend building on these gains and refocusing on deepening our understanding of the commonly used functions, and perhaps even the less commonly used functions, and their interactions, rather than attempting a theory of the nature of argument schemes beyond T1 or T2.

\section{Acknowledgements:}

I thank numerous unknown reviewers of this paper (and earlier versions of this paper) along with audiences at presentations of 
shorter versions of this work, but most especially the audience at the Ninth ISSA conference in Amsterdam in 2018.

\section{References:}

Blair, J. Anthony. 1999. Presumptive reasoning / argument: An overlooked class. Protosociology 13(1): 46-60.

Blair, J. Anthony. 2000. A theory of normative reasoning schemes. In Proceedings of the third OSSA conference: Argumentation at the century's turn, eds. Hans V. Hansen, Christopher W. Tindale, and Elmar Sveda. St. Catharines, ON: OSSA.

Blair, J. Anthony. 2001. Walton's argumentation schemes for presumptive reasoning: A critique and development. Argumentation 15(4): 365-379.

Dove, Ian J., Nussbaum, E. Michael. 2018. In Argumentation and inference: Proceedings of the 2nd European conference on argumentation, Fribourg, eds. S. Oswald and D. Maillat, D, (2): 261-278. London: College Publications.

Garssen, Bart. 2001. Argumentation schemes. In Crucial concepts in argumentation theory, ed. Frans H. van Eemeren, 81-99. Amsterdam: University Press.

Garssen, Bart. 1994. Recognizing argumentation schemes. In Studies in Pragma-Dialectics, eds. Frans H. van Eemeren and Rob Grootendorst, 105-111. Amsterdam: Sic Sat.

Goddu, G.C. 2019. A simple theory of argument schemes. Proceedings of the Ninth Conference of the International Society for the Study of Argumentation, Bart J Garssen, et al., (eds.): 421-428. Amsterdam: Sic Sat.

Goddu, G. C. 2016. On the very concept of an enthymeme. OSSA Conference Archive 84. URL:

$<$ http://scholar.uwindsor.ca/ossaarchive/OSSA11/papersandcomment aries/84>

Hitchcock, David. 2017. On reasoning and argument. Cham: Springer International Publishing AG.

Hitchcock, David. 1998. Does the traditional treatment of enthymemes rest on a mistake? Argumentation 12(1): 15-37. (Reprinted in Hitchcock (2017).

Hoppman, Michael J. 2018. Towards a chemical taxonomy for types of arguments and inferences. Argumentation and inference: Proceedings of the 2nd European conference on argumentation, Fribourg, 
eds. S. Oswald and D. Maillat, D, (1): 593-605. London: College Publications.

Katzav, Joel, Reed, Christopher. 2004. On argumentation schemes and the natural classification of arguments. Argumentation 18(2): 239259.

Kienpointner, Manfred. 1992. How to classify arguments. In Argumentation illuminated, eds. F. van Eemeren, R. Grootendorst, A. Blair, and C. Willard, 178-188. Amsterdam: University Press.

Kienpointner, Manfred. 2018. Thirty years after: Towards a typology of argument schemes. In Argumentation and inference: Proceedings of the 2nd European conference on argumentation, Fribourg, eds. S. Oswald and D. Maillat, D, (1): 543-558. London: College Publications.

Keller, Lorraine. 2013. The metaphysics of propositional constituency. Canadian journal of philosophy 43(5-6): 655-678.

Lumer, Christopher. 2011. Argument schemes - an epistemological approach. In Argumentation: Cognition \& community. Proceedings of the 9th OSSA conference, ed. Frank Zenker. CD-ROM, 1-32. Windsor, ON: OSSA.

Macagno, Fabrizio. 2015. A means-end classification of argumentation schemes. In Reflections on theoretical issues in argumentation theo$r y$, eds. F. van Eemeren and B. Garssen, 183-201. Cham: Springer.

Macagno, Fabrizio. 2018. Classifying and combining argumentation schemes for representing real arguments. In Argumentation and inference: Proceedings of the 2nd European conference on argumentation, Fribourg, eds. S. Oswald and D. Maillat, D, (2): 559-576. London: College Publications.

Pinto, Robert C. 2003. Commentary on C. Reed and D. Walton 'Argumentation schemes in argument-as-process and argument-asproduct.' In Informal Logic at 25: Proceedings of the Windsor conference, Eds. J.A. Blair, D. Farr, H.V. Hansen, R.H. Johnson and C.W. Tindale, Windsor, ON: OSSA. URL: $<$ https://scholar.uwindsor.ca/ossaarchive/OSSA5/papersandcomment aries/76/>

Wagemans, Jean H.M. 2018. Assertoric syllogistic and the periodic table of arguments. In Argumentation and inference: Proceedings of the 2nd European conference on argumentation, Fribourg, eds. S. Oswald and D. Maillat, D, (1): 577-591. London: College Publications.

Walton, Douglas N., C. Reed and F. Macagno. 2008. Argumentation schemes. Cambridge: Cambridge University Press. 
Walton, Douglas N., Christopher Reed. 2005. Argumentation schemes and enthymemes. Synthese, 145(3): 339-370. URL: $<$ http://doi.org/10.1007/s11229-005-6198-x>

Walton, Douglas N. 1996. Argument schemes for presumptive reasoning. Mahwah: Lawrence Erlbaum.

Walton, Douglas N., Godden, David. 2005. The nature and status of critical questions. In The uses of argument: Proceedings of a conference at McMaster University, ed. D. Hitchcock, 476-484. Hamilton, Ontario: OSSA.

Walton, Douglas N., Godden, David. 2007. Advances in the theory of argumentation schemes and critical questions. Informal logic, 27(3): 276-292.

Wyner, Adam. 2016. A functional perspective on argumentation schemes. Argument \& computation 7(2-3): 113-133. 